\title{
Experimantal oberving of transformation Bessel beam spreading along axis of crystal during wavelength changes
}

\author{
V.S. Vasilev ${ }^{1}$, V.V. Podlipnov ${ }^{1,2}$ \\ ${ }^{1}$ Samara National Research University, 34 Moskovskoe Shosse, 443086, Samara, Russia \\ ${ }^{2}$ Image Processing Systems Institute - Branch of the Federal Scientific Research Centre "Crystallography and Photonics" of Russian Academy of Sciences, 151 \\ Molodogvardeyskaya st., 443001, Samara, Russia
}

\begin{abstract}
In paper decribe experimental observing transform bessel beam, formed by diffraction axicon in moment propagation through anisortopic birefringence crystal. This observation covers large range wavelength changes (from $520 \mathrm{~nm}$ to $534 \mathrm{~nm}$ ). Theoretical explain effect is given.
\end{abstract}

Keywords: laser with changing wavelength; diffraction axicon; birefringent crystal; bessel beams

\section{Introduction}

It is well-known the usage of anisotrophic elements to convert beams with the homogeneous polarization into cylindrical vector beams [1-6]. At the same time, it is necessary to implement the separation of the longitudinal modes along the optical axis of the system, which is parallel to the axis of the crystal. To improve convergence of the beams in the crystal, it is possible to use telescopic system or to form beams with high numerical aperture. Polarization and mode conversion during propagation along the axis of the crystal were considered for both Bessel and Gaussian beams [7-16].

It has been shown in the studies $[17,18]$ that during the propagation along the crystal axis neuraxial Bessel beams have other properties than Gaussian beams, namely, experiencing a uniform periodic change of intensity. In this case, the Bessel beam of zero order and second-order are periodically converted from one to another [7-9, 17, 18]. The oscillation period is directly proportional to the wavelength of the laser radiation and inversely proportional to the square of the spatial frequency of the laser beam and the difference of the dielectric capacitivity, which is corresponding to the ordinary and extraordinary rays. This dependence allows control occurring transformation in the crystal due to changes of the characteristics in either Bessel beam or crystal. In particular, the spatial frequency of the beam depends on the numerical aperture of the axicon [19-22] which shapes the beam, also it is possible to adjust characteristics of the beam by changing the beam divergence [23]. To change the parameters of the crystal, it can be heated [24] or effected by electro-optic [25]. However, the most convenient way of adjustment is to change the wavelength of the laser radiation which has a direct linear relationship from the period of transformation [26].

It was experimentally demonstrated the ability [26] to manage the transformation of the Bessel beam at the output of the $\mathrm{CaCO} 3$ crystal by changing the wavelength of the radiation illuminating the diffractive axicon. It was achieved almost complete transformation of the Bessel beam of zero order beam to the second order using the axicon period of $2 \mu \mathrm{m}$ and the wavelength at $\Delta \lambda=1.5$ of the initial value of $\lambda=637.5$. The variation of the wavelength within a small range was achieved by changing the temperature of the laser. In contrast to this method, the usage of a laser with variable wavelength provides a wide range of $\Delta \lambda$, and therefore the possibility of achieving complete conversion using the axicon with a large period, i.e., a smaller numerical aperture. Note that the usage of axicons with high numerical aperture is limited not only with technological possibilities of production [27] and reduction of non-diffraction distribution cut [20], but with the limiting numerical aperture [28], in which propagating waves occur in the considered optical medium.

This paper shows the results of experimental observation of the mode conversion of Bessel beam formed by the axicon amplitude with a period of $3 \mu \mathrm{m}$ with the output of a deuterated potassium dihydrogen phosphate crystal when the wavelength of the laser EKSPLA NT 200 radiation is changed.

\section{Theoretical analysis}

Consider an anisotrophic crystal whose axis is oriented along the optical axis.

The intensity distribution $\mathrm{I}(\mathrm{x}, \mathrm{y}, \mathrm{z})$ in the propagation of Bessel beam along the axis of the crystal is as follows [9, 11, 17, 18]:

$$
\begin{aligned}
& I(x, y, z) \approx \frac{1}{2}|C(z)| J_{0}^{2}\left(k \sqrt{x^{2}+y^{2}}\right)^{+}|S(z)|^{2} J_{2}^{2}\left(k \sqrt{x^{2}+y^{2}}\right) \\
& \text { where } J_{0}(\cdot){ }_{\text {и }}^{2} J_{2}(\cdot)-\text { Bessel functions of zero and second order, respectively, } \\
& C(z)=\exp \left(i k z \gamma_{o}\right)+\exp \left(i k z \gamma_{e}\right) \\
& S(z)=\exp \left(i k z \gamma_{o}\right)-\exp \left(i k z \gamma_{e}\right)
\end{aligned}
$$

where $\alpha$ - numerical aperture of the beam, $\mathrm{z}$ - is the distance traveled; $\gamma_{\mathrm{o}}$, $\gamma_{\mathrm{e}}$ - are the values which are determining the direction of propagation of the ordinary and extraordinary rays: 
$\gamma_{o}=n_{o}^{2}-\alpha^{2}$

$\gamma_{e}^{o}=\sqrt{c_{o}^{2}-\alpha^{2} n_{o e}^{2} / n^{2}}$

where no, ne - ordinary and extraordinary refractive indices of the crystal.

A complete transformation of the Bessel beam of zero order to the beam of second order will periodically occur at distances that are multiples of the value:

$$
z_{p}=\frac{\lambda}{\gamma_{o}-\gamma_{e}} \approx \frac{2 \lambda n \underset{o e}{n^{2}}}{\alpha^{2}\left(n_{o}{ }^{2}-n_{e}{ }^{2}\right)}
$$

Full transformation period depends on the refractive indices of the crystal and the numerical aperture of the axicon, as well as on the wave length of radiation. Moreover, the wavelength dependence is direct and linear, i.e. the most convenient to dynamically change the value of period so that the output of the crystal is formed the desired pattern.

\section{Experimental results}

\subsection{Method of experiment}

In this paper experiments were conducted using the optical arrangement shown in the fig.1, where 1 - laser with changing wavelength EKSPLA NT 200, 2 - diaphragm, 3 - collimator, 4 - diaphragm, 5 - DOE, 6 - anisotropic crystal, 7 - 20x microobjective, 8 - digital USB camera TOUPCAM UCMOS05100KPA.

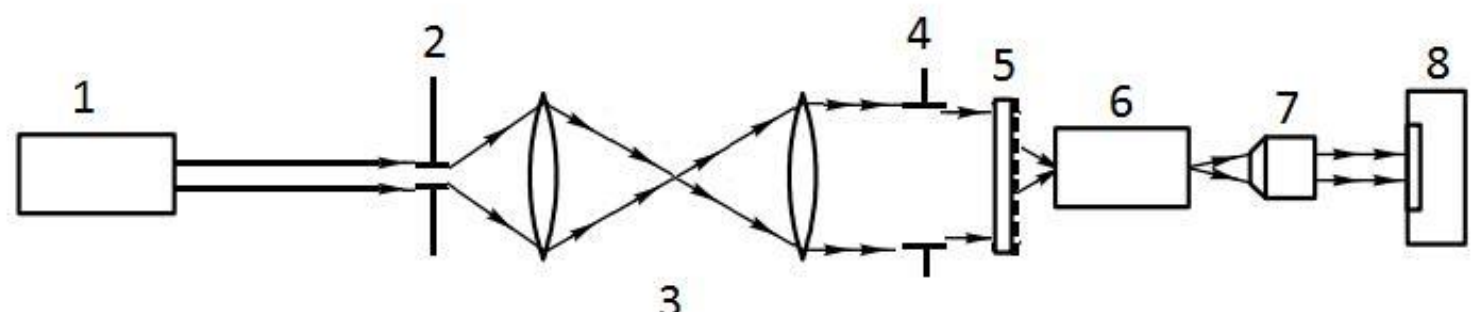

3

Fig. 1. Optical setup of the experiment.

A laser with variable wavelength was used as a radiation source EKSPLA NT 200. In the considered range of wavelength variation $(520-534 \mathrm{~nm})$, the laser beam has a horizontal X-polarization. The energy of the laser radiation obtained in the range of the visible spectrum wavelength is variable from 610 microjoule $(450 \mathrm{~nm})$ to 45 microjoule $(700 \mathrm{~nm})$. The extension of the beam is done by the collimator. Owing to the fact that the beam emerging from the laser has a Gaussian intensity distribution, it has become necessary to select a part of the beam with a small change of intensity. This problem can be solved by introduction of a diaphragm 2. Septum 4 allows to limit the numerical aperture and to enable formation of the propagating waves. The intensity distribution of the output beam was recorded with a digital USB camera with a resolution of 5 mega pixels and ADC digit capacity of 12 bits.

The Bessel beam of zero order is formed by using a diffraction amplitude of the axicon with period which operates with nearly the same effectiveness in the considered wavelength range. The Bessel beam was directed along the axis of a crystal with cross-sectional dimension and length $20 \mathrm{~mm}$. As a result of Bessel beams transformation there were formed interference pattern intensity distribution for different wavelengths and it was recorded with the microscope objective and digital cameras (table 1). To highlight different $\mathrm{X}$ and $\mathrm{Y}$ components of the transformed beams a rotating analyzer was installed in front of the digital camera.

\subsection{Results and discussion}

As you can see in the images, when the wavelength changes by $\Delta \lambda=14 \mathrm{~nm}$ there is a complete transformation of the Bessel beam of the first order to the second, which is caused by the reaction of doubly refracting crystal. The observed phenomenon is explained by the formula (4), where $\lambda$ is in the numerator. At the same time, change of wavelength is similar to the changes of the propagation length of the beam, as if it had been changed the dimensions of the crystal. To verify the observed phenomenon in the described conversion model it was carried out an additional numerical calculation for the wavelength of $520 \mathrm{~nm}$ and 532 $\mathrm{nm}$. Intensity distributions of the Bessel beams images which were converted by electro-optic crystal for given experimental conditions obtained by numerical calculation are presented in table 2 . 
Computer Optics and Nanophotonics / V.S. Vasilev, V.V. Podlipnov

Based on the simulation results, we can conclude that the observed experimental results are very similarity with the mathematical description for the Bessel beams conversion in the considered wavelength range.

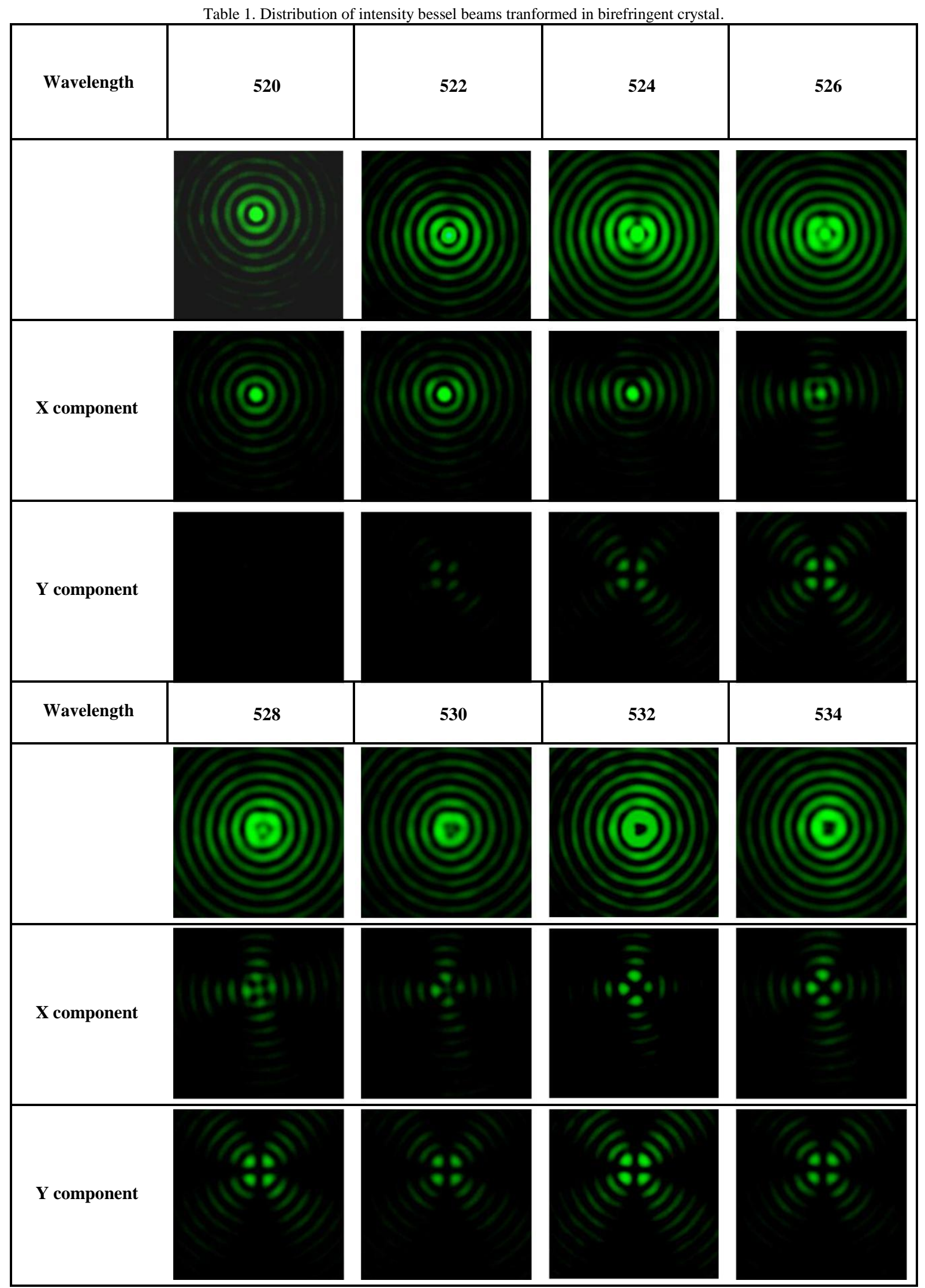


Table 2. Modeling distribution of intensity bessel beams transformed in birefringent crystal.

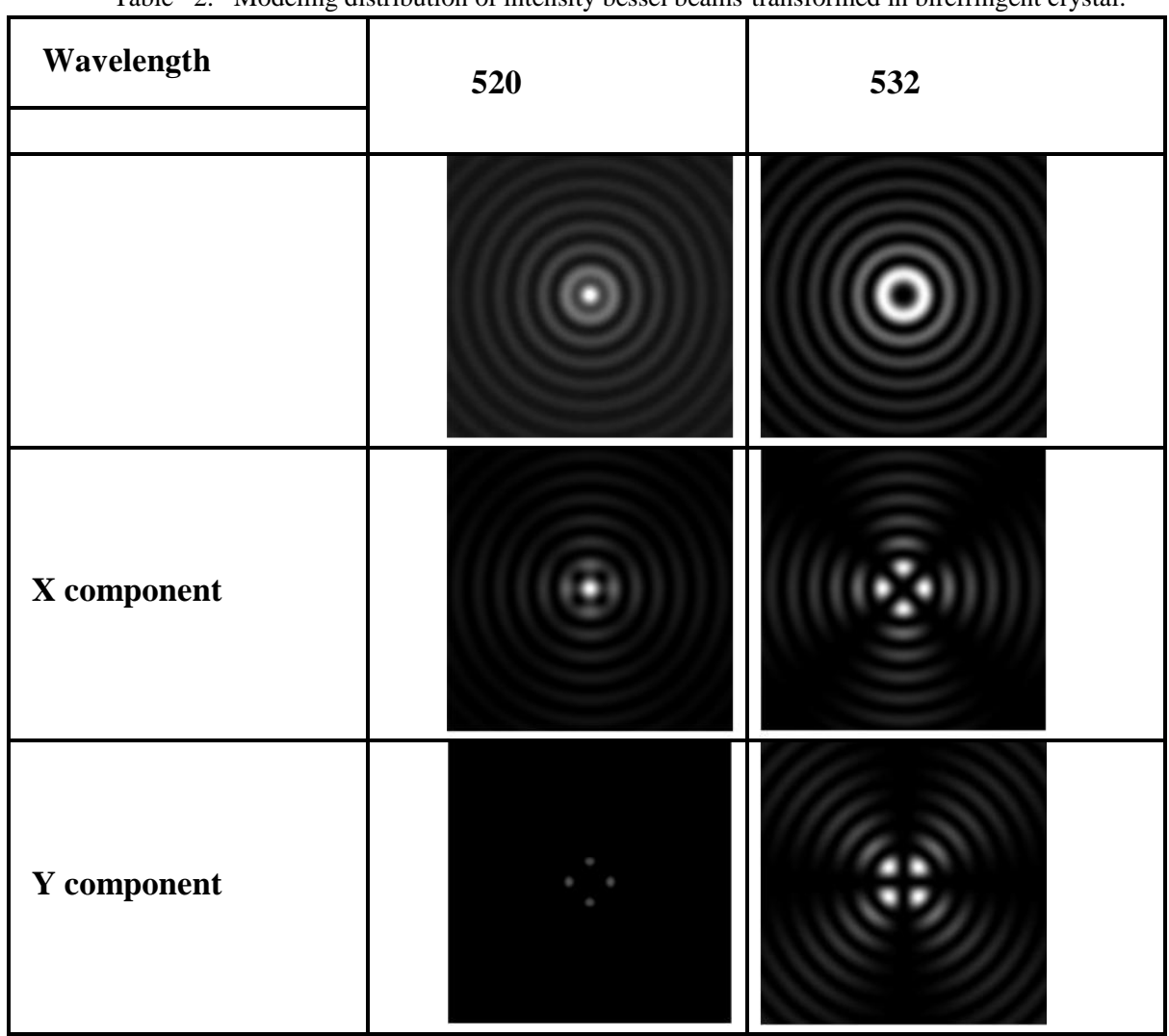

\section{Conclusion}

It was experimentally demonstrated the conversion of Bessel beams of zero order, generated by the axicon with period in birefringence crystal, depending on the change of the wavelength of the laser radiation in the range of $\lambda=520-534 \mathrm{~nm}$ to the Bessel beams of the second order, which has an annular intensity distribution. Further increase of the wavelength has showed a recurrent re-transformation into a Bessel beam of zero order. Comparative analysis of experimental images of full intensity and their components with images obtained by the numerical simulation has showed their similarity.

\section{Asknowledgement}

This work was supported by the Ministry of Education, by the Russian Foundation for Basic Research (grant 16-29-11698 ofi_m and 16-07-00494 a) and by the grant from the President of the Russian Federation to support young Russian scientists-doctors of science, (project no. MD- 5205.2016.9).

\section{References}

[1] Machavariani G, Lumer Y, Moshe I, Meir A, Jackel S, Davidson N. Birefringence-induced bifocusing for selection of radially or azimuthally polarized laser modes. Applied Optics 2007; 46(16): 3304.

[2] Yonezawa K, Kozawa Y, Sato S. Compact laser with radial polarization using birefringent laser medium. Journal of Applied Physics 2007; 1(1): 5160.

[3] Zhan Q. Cylindrical vector beams: from mathematical concepts to applications. Advances in Optics and Photonics 2009 ; $1(1)$ : 57.

[4] Fadeyeva T, Shvedov V, Shostka N, Alexeyev C, Volyar A. Natural shaping of the cylindrically polarized beams. Optics Letters $2010 ; 235$ (22): 3787.

[5] Khonina SN, Karpeev SV, Alferov SV. Theoretical and an experimental research of polarizing transformations in uniaxial crystals for generation cylindrical vector beams of high orders. Computer Optics 2014; 38(2): 171-180.

[6] Khonina SN, Karpeev SV, Alferov SV, Soifer VA. Generation of cylindrical vector beams of high orders using uniaxial crystals. Journal of Optics 2015; 17(1): 11.

[7] Khilo NA, Ryzhevich AA, Petrova ES. Transformation of the order of Bessel beams in uniaxial crystals. Quantum Electronics 2001; 31(1): 85-89.

[8] Khilo NA. Diffraction and order conversion of Bessel beams in uniaxial crystals. Optics Communications 2012; 285(1): 503-509.

[9] Khonina SN, Morozov AA, Karpeev SV. Effective transformation of a zero-order Bessel beam into a second-order vortex beam using a uniaxial crystal. Laser Phys. 2014: 24(1): 5.

[10] Khonina SN, Paranin VD, Ustinov AV, Krasnov AP. Astigmatic transformation of Bessel beams in a uniaxial crystal. Optica Applicata 2016; Vol. 46(1): $5-18$.

[11] Khonina SN, Karpeev SV, Morozov AA, Paranin VD. Implementation of ordinary and extraordinary beams interference by application of diffractive optical elements. Journal of Modern Optics 2016; 63(13): 1239-1247.

[12] Ciattoni A, Cincotti G, Palma C. Circularly polarized beams and vortex generation in uniaxial media. J. Opt. Soc. Am. A $2003 ; 20(1)$ : $163-171$.

[13] Marrucci L, Manzo C, Paparo D. Optical spin-to-orbital angular momentum conversion in inhomogeneous anisotropic media. Phys. Rev. Lett. 2006; 96(1): 130-135.

[14] Loussert C, Brasselet E. Efficient scalar and vectorial singular beam shaping using homogeneous anisotropic media Optics Letters 2010; 35(1): 7-9. 
[15] Fadeyeva TA, Shvedov VG, Izdebskaya YV, Volyar AV, Brasselet E, Neshev DN, Desyatnikov AS, Krolikowski W, Kivshar YS. Spatially engineered polarization states and optical vortices in uniaxial crystals. Optics Express 2010; 18(10): 63.

[16] Picon A, Benseny A, Mompart J, Calvo GF. Spin and orbital angular momentum propagation in anisotropic media: theory. J. Opt. 2011 ; $13(1)$ : 7.

[17] Khonina SN, Volotovsky SG, Kharitonov SI. Features of nonparaxial propagation of Gaussian and Bessel beams along the axis of the crystal. Computer Optics 2013; 37(3): 297-306.

[18] Khonina SN, Kharitonov SI. Comparative investigation of nonparaxial mode propagation along the axis of uniaxial crystal. Journal of Modern Optics 2015; 62(2): 125-134.

[19] McLeod, JH. The axicon: a new type of optical element. Journal of the Optical Society of America 1954; 44: $592-597$.

[20] Turunen J, Vasara A, Friberg AT. Holographic generation of diffraction-free beams. J. Appl. Opt. 1988; 27(19): 3959-3962.

[21] Khonina SN, Kotlyar VV. Bessel-mode formers. Proceedings of SPIE 1994; 23(63): 184-190.

[22] Chattrapiban N, Rogers E, Cofield D, Hill W, Roy R. Generation of nondiffracting Bessel beams by use of a spatial light modulator. Opt. Lett. 2003; 28(22): 2183-2185

[23] Paranin VD, Karpeev SV, Khonina SN. Control of the formation of vortex Bessel beams in uniaxial crystals by varying the beam divergence. Quantum Electronics 2016; 46(2): 163-168.

[24] Paranin VD, Khonina SN, Karpeev SV. Control of the optical properties of a CaCO3 crystal in problems of generating Bessel vortex beams by heating. Optoelectronics, Instrumentation and Data Processing 2016; 52(2): 174-179.

[25] Khonina SN, Paranin VD. Electro-optical correction of Bessel beam conversion along axis of a barium niobate-strontium crystal. Computer Optics 2016; 40(4): 475-481. DOI: 10.18287/2412-6179-2016-40-4-475-481.

[26] Paranin VD, Karpeev SV, Khonina SN. Transformation of Bessel beams in c-cuts of uniaxial crystals by varying the emission source wavelength. Journal of Russian Laser Research 2016; 37(3): 207-210.

[27] Cherkashin VV, Kharissov AA, Korol'kov VP, Koronkevich VP, Poleshchuk AG. Accuracy potential of circular laser writing of DOEs. Proceedings of SPIE 1997; 3348: 58-68.

[28] Ustinov AV, Khonina SN. Analysis of laser beam diffraction by axicon with the numerical aperture above limiting. Computer Optics 2014; 38(2): 213222 . 